\title{
Aging affects acquisition and reversal of reward-based associative learning
}

\author{
Julia A. Weiler, ${ }^{1}$ Christian Bellebaum, and Irene Daum \\ Institute of Cognitive Neuroscience, Department of Neuropsychology, Ruhr-University of Bochum, 44780 Bochum, Germany
}

\begin{abstract}
Reward-based associative learning is mediated by a distributed network of brain regions that are dependent on the dopaminergic system. Age-related changes in key regions of this system, the striatum and the prefrontal cortex, may adversely affect the ability to use reward information for the guidance of behavior. The present study investigated the effects of healthy aging on different components of reward learning, such as acquisition, reversal, effects of reward magnitude, and transfer of learning. A group of 30 young (mean age $=24.2 \mathrm{yr}$ ) and a group of 30 older subjects (mean age $=64.1 \mathrm{yr}$ ) completed two probabilistic reward-based stimulus association learning tasks. Older subjects showed poorer overall acquisition and impaired reversal learning, as well as deficits in transfer learning. When only those subjects who showed evidence of significant learning were considered, younger subjects showed equivalently fast learning irrespective of reward magnitude, while learning curves in older subjects were steeper for high compared to low reward magnitudes. Acquired equivalence learning, which requires generalization across stimuli and transfer of learned contingencies to new stimuli, was mildly impaired in older subjects.
\end{abstract}

Rewards play a critical role in the guidance of goal-directed behavior, with detection, evaluation, and prediction of rewards being important determinants. Reward processing is mediated by the dopaminergic system and a network involving midbrain areas, the striatum, amygdala, medial temporal, and orbitofrontal cortex (OFC), as well as several neocortical regions (Schultz 2000). The striatum, the prefrontal cortex (PFC), and the medial temporal lobe (MTL) are known to undergo substantial changes during the course of normal aging (Gunning-Dixon et al. 1998; Raz and Rodrigue 2006). The most pronounced volume reductions are seen in the PFC, while the striatum and the MTL region are somewhat less affected (Tisserand and Jolles 2003; Hedden and Gabrieli 2004). In addition, age-related loss of dopamine receptors and transporters in striatal and extrastriatal regions may adversely affect the functional integrity of the reward learning system (Bäckman et al. 2006). While it is clear that transmitters other than dopamine are also involved in reward processing (Beninger and Gerdjikov 2004), dopamine is commonly regarded as the most important one.

So far, relatively little is known about the influence of healthy aging on reward-based stimulus association learning. There is evidence of age-related deficits of reversal learning in animals, although sparing of function has also been reported (Rapp 1990; Lai et al. 1995; Herndon et al. 1997; Voytko 1999; Schoenbaum et al. 2002; Tapp et al. 2003). In a study of human aging effects, Mell et al. (2005) reported impairments of older relative to young adults on a reward-based probabilistic object reversal task, presumably linked to reduced ventral striatal activation in the older subjects (Marschner et al. 2005). Performance on another reward-related task, the Iowa Gambling task, which has been linked to OFC function, seems to be, however, not consistently affected by age, being compromised only in a subset of older subjects (MacPherson et al. 2002; Denburg et al. 2005). Two recent neuroimaging studies compared the brain activations during reward processing between young and aged individuals. Larkin et al. (2007) did not observe significant age effects on striatal and insular activations during monetary gain anticipa-

\footnotetext{
1Corresponding author.
}

E-mail julia.weiler@rub.de; fax 49-234-32-14622.

Article is online at http://www.learnmem.org/cgi/doi/10.1101//m.890408. tion, but an age-related reduction of activation during loss anticipation. Data by Schott et al. (2007), however, suggested that unlike young subjects, older people do not exhibit ventral striatal and midbrain activation during reward anticipation, but during the reward feedback itself. The authors concluded that, although general reward processing might be intact in older people, they are impaired in learning the predictive value of the stimuli.

The human striatum was shown to be involved in reward processing by several functional neuroimaging studies (Delgado et al. 2000, 2003; Elliott et al. 2000, 2003; O'Doherty 2004; Delgado 2007). It has, however, also been suggested that rewardassociated striatal activations reflect the coding of stimulus salience rather than responses to rewards (Zink et al. 2004). Alternatively, the dopaminergic midbrain and its striatal target regions may play a more general role in performance feedbackbased learning and not specifically in reward-based associative learning (Aron et al. 2004), a view that is consistent with probabilistic learning deficits in Parkinson's disease patients (Knowlton et al. 1996; Myers et al. 2003; Shohamy et al. 2004). The striatum has recently been found to be involved in coding expected values (Knutson et al. 2005) and in the distinction between valences and magnitudes of rewarding outcomes (Delgado et al. 2003). Magnitude- and valence-dependent activity is commonly observed in the OFC (O'Doherty et al. 2001; Elliott et al. 2003; Wallis and Miller 2003; Roesch and Olson 2004; Wallis 2007). The OFC is also important for performance associated with reversal learning, presumably in cooperation with the anterior cingulate cortex (Rolls 2000; Kringelbach 2005). The dorsolateral PFC, on the other hand, is assumed to use reward information to plan and execute behavior directed toward rewards (Schultz 2000). Furthermore, during the course of performance feedback-based associative learning, MTL activations were observed early during learning, followed by later striatal activations (Poldrack et al. 2001). Additionally, generalization and transfer learning as assessed by acquired equivalence paradigms are predominantly dependent on MTL function (Myers et al. 2003; Keri et al. 2005).

Since aging leads to several changes in the brain regions linked to the reward system, the present study aimed to determine the functional sequelae of normal aging on a range of cognitive processes involved in probabilistic reward-based learning 
paradigms. Two different reward-based learning tasks were administered to a group of young and a group of older participants. Subjects had to learn the associations between four Asian symbols and two colors. On each trial, they were presented with one of the symbols and subsequently had to select one of two colors (see Fig. 1A). Correct responses were rewarded with monetary feedback of either 5 or 20 cents; however, feedback was only given on $80 \%$ of all trials (probabilistic learning). In the first task, the slow acquisition of stimulus associations was followed by a reversal of contingencies. Subsequently, contingencies were changed back to the initial schedule of the acquisition phase. During this learning stage (single symbol reversal), subjects only learned the new associations for two of the symbols and were then tested on all four symbols without receiving trial-by-trial feedback (transfer learning) (see Fig. 1B). The second task was an acquired equivalence paradigm, in which subjects learned associations between new Asian symbols and new colors. In a second phase of this experiment, the colors changed and subjects had to learn the new associations for half of the symbols. As in the first reward-based learning task, the subsequent test phase involved all symbols without response feedback (see Fig. 1C). While performance on the first learning task is assumed to rely predominantly on striatal and OFC functions, the second task is presumably more closely linked to striatal and MTL functions (Myers et al. 2003; Keri et al. 2005). Subjects also completed a verbal paired associates learning task to assess declarative learning performance, and were screened for general neuropsychological status.

\section{Results}

Short-term and working memory

Short-term and working memory were screened because these abilities might contribute to the successful completion of the reward learning tasks. Analysis of digit spans (forward and backward reproduction) did not yield significant age group differences (both $P>0.130$; see Table 1). In contrast, block spans were consistently shorter in the older group for both forward and backward reproduction (both $P<0.001$; see Table 1 ).

\section{Verbal paired associates}

The results of the verbal paired associates test, which assesses declarative learning performance, are shown in Figure 2. Analysis of variance (ANOVA) with factors Group (younger vs. older subjects), Difficulty (easy vs. hard), and Block (three blocks) yielded main effects of Group $\left(F_{(1,57)}=25.16, P<0.001\right)$, Difficulty $\left(F_{(1,57)}=52.02, P<0.001\right)$, and Block (linear trend: $\left.F_{(1,57)}=115.95, P<0.001\right)$, as well as a significant three-way interaction $\left(F_{(1,57)}=4.70, P=0.034\right)$. The main effects reflect better performance of younger relative to older subjects and better performance for easy relative to hard items, as well as significant learning across blocks. The interaction reflects a larger difference between easy and hard items in the older group at the beginning of learning which becomes smaller across blocks (see Fig. 2).

Analysis of delayed recall indicated that on average, young subjects remembered more hard pairs than older subjects $\left(t_{(57)}=2.98, P=0.004\right.$; see Fig. $\left.2 \mathrm{~B}\right)$, while this was not the case for easy items $\left(t_{(57)}=0.61, P=0.542\right.$; see Fig. $\left.2 \mathrm{~A}\right)$.

\section{Stimulus-reward learning}

The first reward learning task concentrated on the slow acquisition of stimulus contingencies, reversal, and transfer learning. Data for acquisition and reversal were analyzed by ANOVA with factors Group (younger vs. older subjects), Block (three blocks of 40 trials each), and Reward Magnitude (5 vs. 20 cents). Analysis of acquisition yielded main effects of Group $\left(F_{(1,58)}=28.15\right.$, $P<0.001$ ), Block (linear trend: $F_{(1,58)}=47.89, P<0.001$ ), and Reward Magnitude $\left(F_{(1,58)}=6.33\right.$, $P=0.015)$; none of the interactions
A

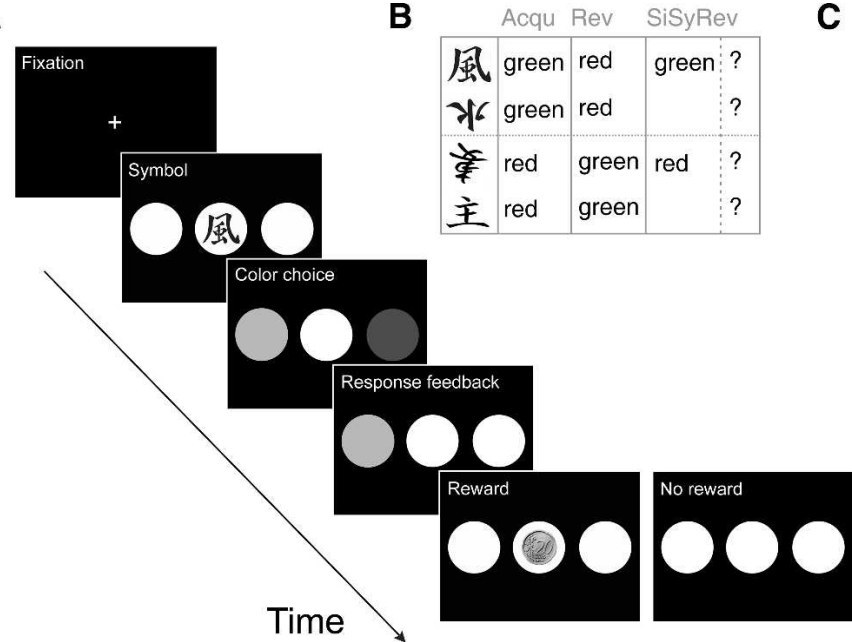

\begin{tabular}{|c|c|c|c|}
\hline & Acqu1 & Acqu2 & Test \\
\hline 氣 & brown & yellow & $?$ \\
\hline 覓 & brown & & ? \\
\hline 陽 & pink & blue & ? \\
\hline 爱 & pink & & ? \\
\hline
\end{tabular}
reached significance (all $P>0.160$ ). Older subjects showed poorer learning than younger subjects, learning was generally better with higher compared to lower rewards, and performance of both groups improved significantly over the blocks (Fig. 3A).

For reversal, ANOVA revealed main effects of Group $\left(F_{(1,58)}=13.70, P<0.001\right)$ and Block (linear trend: $F_{(1,58)}=31.85$, $P<0.001)$, as well as a significant Block $\times$ Group interaction (linear trend: $\left.F_{(1,58)}=9.01, P=0.004\right)$, but no significant effects involving Reward Magnitude (main effect: $F_{(1,58)}=0.56$, $P=0.459$ ). The younger group showed significant learning across blocks (linear trend: $\left.F_{(1,29)}=42.10, P<0.001\right)$, while the Block effect did not reach significance in the older group (linear trend: $F_{(1,29)}=3.14, P=0.087$ ) (see Fig. 3A).

To further assess age group differences, the proportions of learners and non-learners were determined as a measure of reward learning. Learners were defined as subjects who achieved at least $70 \%$ correct responses in the last block of the acquisition phase for at least one of the two reward magnitudes. The probability that this score is obtained by chance is lower than $6 \%$. The $70 \%$ crite- 
Table 1. Sample description

\begin{tabular}{lrrr}
\hline & \multicolumn{1}{c}{ Young } & \multicolumn{1}{c}{ Old } & \\
\hline & Mean (SD) & Mean (SD) & \multicolumn{1}{c}{$\boldsymbol{P}$} \\
\hline Age (years) & $24.2(2.8)$ & $64.1(6.2)$ & $<0.001$ \\
Years of education & $13.0(0.0)$ & $10.1(1.8)$ & $<0.001$ \\
IQ & $112.3(6.9)$ & $117.4(9.2)$ & 0.017 \\
Digit span forward & $9.9(1.1)$ & $9.2(2.1)$ & 0.156 \\
Digit span backward & $8.4(2.4)$ & $7.5(2.3)$ & 0.134 \\
Block span forward & $9.4(1.6)$ & $7.6(1.6)$ & $<0.001$ \\
Block span backward & $9.7(1.4)$ & $6.8(1.6)$ & $<0.001$ \\
\hline
\end{tabular}

Results of the digit and block span tasks are given by means of raw scores.

rion reflects a high level of learning since in the probabilistic setup, application of the underlying rule would only lead to a maximum score of $80 \%$. Applying this criterion, 25 of the 30 younger subjects, but only 17 of the 30 older subjects, were classified as learners, the proportion of learners being significantly higher in the younger group $\left(\chi^{2}\right.$ test: $\chi^{2}=5.08$, d.f. $=1$, $P=0.024)$. Learners and non-learners did not differ significantly on any of the non-reward learning measures, with the exception of the sum score for verbal paired associates learning (immediate recall) being higher in the learners $\left(t_{(1,57)}=2.39, P=0.020\right)$.

As reversal learning is dependent on previous acquisition, further analyses were limited to the subgroup of subjects who showed successful initial learning. Additionally, this procedure allowed the analysis of the effect of reward magnitude on the course of learning in subjects who actually showed significant learning. Learning curves for the learners are presented in Figure 3B. For the acquisition phase, there were significant main effects of Group $\left(F_{(1,40)}=13.74, P=0.001\right)$, Block (linear trend: $\left.F_{(1,40)}=77.58, P<0.001\right)$, and Reward Magnitude $\left(F_{(1,40)}=8.58\right.$, $P=0.006)$, as well as a trend toward a significant three-way interaction (Group $\times$ Block [linear trend] $\times$ Reward Magnitude, $\left.F_{(1,40)}=3.91, P=0.055\right)$. Exploratory ANOVA in the young learners yielded main effects of Block (linear trend: $F_{(1,24)}=41.24$, $P<0.001)$, and Reward Magnitude $\left(F_{(1,24)}=11.72, P=0.002\right)$, but no interaction (linear trend: $F_{(1,24)}=0.12, P=0.890$ ). A separate analysis for older learners yielded a main effect of Block (linear trend: $\left.F_{(1,16)}=44.54, P<0.001\right)$ and a trend toward a significant Block $\times$ Reward Magnitude interaction $\left(F_{(1,16)}=4.15, P=0.059\right)$. Further analyses indicated that older subjects showed faster learning across blocks for the symbols rewarded with 20 cents $\left(F_{(1,16)}=41.78, P<0.001\right)$ compared to those rewarded with 5 cents $\left(F_{(1,16)}=5.79, P=0.026\right)$. The pattern of results for the

A Easy items

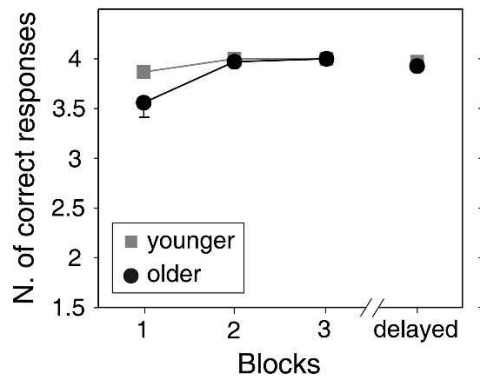

Figure 2. Results of the verbal paired associates test for the three immediate retrieval blocks and the delayed recall separately shown for easy $(A)$ and hard $(B)$ items. The maximum number (N.) of correct responses was 4 for both item categories. Error bars represent standard errors. Note that missing error bars represent standard errors that are too small to be visible with this scaling. analyses of the reversal phase in the subgroup of learners was identical to the pattern of results for all subjects described above.

For the analysis of the single symbol reversal, only subjects who showed significant learning during initial acquisition and who reached the learning criterion in the acquisition phase of the single symbol reversal (five correct successive responses) were included. Using these criteria, 21 out of 30 younger subjects and 14 out of 30 older subjects were defined as learners, indicating a trend toward a higher proportion of learners in the younger group ( $\chi^{2}$ test: $\chi^{2}=3.36$, d.f. $=1, P=0.067$ ).

Data of the single symbol reversal test stage are separated into responses to symbols already presented in the learning stage (learned symbols) and symbols only presented in the test stage (transfer symbols). ANOVA with factors Symbol Type (learned vs. transfer) and Group (younger vs. older subjects) yielded a significant effect of Symbol Type $\left(F_{(1,33)}=28.74, P<0.001\right)$, but no significant Group effect $\left(F_{(1,33)}=1.47, P=0.235\right)$ or interaction $\left(F_{(1,33)}=0.29, P=0.596\right)$. There were more correct responses with learned compared to transfer symbols (see Fig. 4A). As can be seen from Figure $4 \mathrm{~A}$, performance was above the chance level for learned symbols in both groups (both $P<0.001$ ), but learning with the transfer symbols in the older group was not significant $\left(t_{(13)}=0, P=1\right)$ and only approached significance in the younger group $\left(t_{(20)}=1.82, P=0.084\right)$.

\section{Response times and misses}

In order to investigate whether the lower performance of the older subjects might be related to an age-dependent motor deficit, response times (RTs) and the number of misses were analyzed for acquisition and reversal. ANOVA of RTs with factors Group, Block, and Reward Magnitude yielded significant Group $\left(F_{(1,58)}=8.55, P=0.005\right)$ and Block effects (linear trend: $\left.F_{(1,58)}=4.24, P=0.044\right)$ for acquisition and a significant Group effect $\left(F_{(1,58)}=4.93, P=0.030\right)$ for reversal. RTs became shorter over the course of acquisition and were generally longer in the older than in the younger group (see Table 2). It should be noted, however, that the quantitative RT differences were very small.

The number of misses, that is, trials in which subjects did not respond, was $0.4 \%(\mathrm{SD}=0.5)$ in the younger group and $1 \%$ $(\mathrm{SD}=1)$ in the older group across all conditions of the experiment. This difference was significant $\left(t_{(1,58)}=-3.13, P=0.003\right)$, but very small in quantitative terms.

\section{Acquired equivalence}

In contrast to the single symbol reversal of the first reward learning task, the acquired equivalence task required generalization across the stimulus material and could not be completed by memory of stimulus contingencies from earlier phases of the experiment. Data are separated into symbols presented in all experimental phases (learned symbols) and symbols only presented in the first acquisition and the test phase (transfer symbols).

All subjects who reached the learning criteria of eight correct choices in a row during acquisition phase 1 (i.e., initial acquisition of stimulus contingencies; brown and pink colors) and five correct successive responses in acquisition phase 2 (acquisition of new stimulus contingencies for two of the symbols, i.e., the learned symbols; yellow and blue colors) were defined as learners in the acquired equivalence task. All 30 younger subjects, but only 15 of the 29 older subjects (missing data in one older subject), were classified as learners, yielding a significant group difference $\left(\chi^{2}\right.$ test: $\chi^{2}=18.99$, d.f. $\left.=1, P<0.001\right)$.

Younger and older learners differed significantly in the number of trials to criterion in acquisition phase $1\left(t_{(43)}=-2.66\right.$, $P=0.011$ ), with older subjects needing more trials ( $m e a n=51.5$, $\mathrm{SD}=11.1$ ) to reach criterion than younger subjects (mean $=43.8$, $\mathrm{SD}=8.0)$. ANOVA of correct responses in the test phase with 
A All subjects

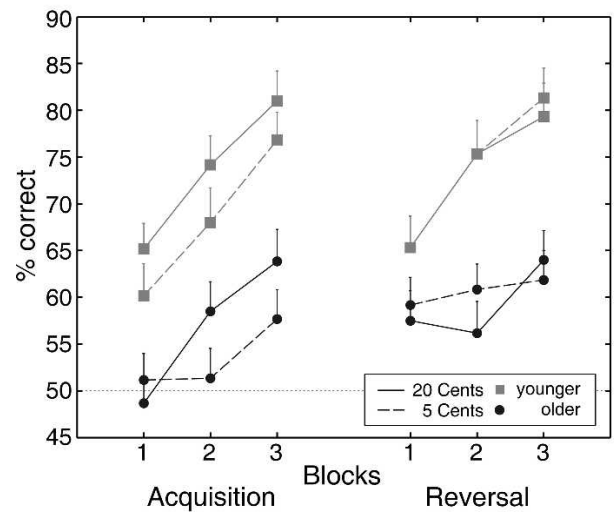

B Learners

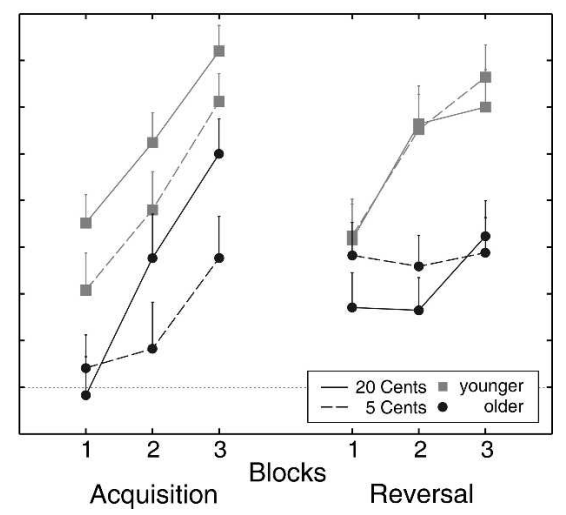

Figure 3. Learning curves for acquisition and reversal phases of the first reward learning task for all subjects $(A)$ and subjects reaching a certain learning criterion in the acquisition phase only $(B)$. The dotted line indicates the chance level; error bars represent standard errors.

factors Group (younger vs. older subjects) and Symbol Type (learned vs. transfer) yielded main effects of Group $\left(F_{(1,43)}=11.49, P=0.002\right)$ and Symbol Type $\left(F_{(1,43)}=5.50\right.$, $P=0.024)$; the interaction was not significant $\left(F_{(1,43)}=0.07\right.$, $P=0.796)$. There were more correct choices for learned compared to transfer symbols, and performance was generally better in younger compared to older subjects (see Fig. 4B). While performance was clearly above the chance level for learned symbols in both groups and transfer symbols in the younger group (all $P<0.020)$, older subjects only showed a strong trend toward performance above chance level for transfer symbols $\left(t_{(14)}=2.12\right.$, $P=0.052)$.

\section{Correlations}

To assess the relationship between neuropsychological and reward learning measures, correlations were calculated between digit and block span scores, verbal paired associates scores (total number of correct responses across all blocks), and performance during acquisition and reversal (percent correct responses), the effect of reward magnitude in acquisition and reversal (performance difference between 5 and 20 cent trials), as well as performance for learned and transfer symbols during single symbol reversal and the acquired equivalence task (percent correct responses). Only correlations significant at the 0.01 level are considered.

In older subjects, visuospatial working memory (block span backward reproduction) correlated significantly with performance during acquisition $(r=0.500, P=0.006)$ and performance for transfer symbols in the acquired equivalence task $(r=0.571$, $P=0.002$ ). There were no significant correlations in the group of younger subjects.

Analyses of intercorrelations of the different reward-based learning measures (percent correct responses in acquisition, reversal, single symbol reversal [separately for learned and transfer symbols], and acquired equivalence [separately for learned and transfer symbols]) yielded significant correlations between acquisition and reversal measures in the younger subjects $(r=0.500$, $P=0.005)$ and between performance for learned and transfer symbols in the acquired equivalence task $(r=0.555, P=0.001)$. In the older group, only the correlation between performance during acquisition and performance with learned symbols in the single symbol reversal reached significance $(r=0.574, P=0.001)$.

\section{Discussion}

The findings of the present study indicate a differential effect of normal aging on different processes involved in reward-based cits since the RTs of older participants were well within the time limit for responding and the number of misses was generally very low in both age groups.

Reward magnitude affected learning in both groups, but there was some evidence of a differential effect on younger and older subjects, when only those subjects who showed evidence for significant learning were considered: Younger subjects showed better overall performance on trials rewarded with 20 cents compared to trials rewarded with 5 cents, while the learning curves were similar. Older subjects, on the other hand, showed a steeper learning curve for trials rewarded with the higher reward magnitude. Reward magnitude had no significant effect in the reversal phase. While the impairments of older adults during acquisition and reversal were significant whether the data were analyzed for all subjects or for learners only, it has to be pointed out that the single symbol reversal and acquired

\section{A Single symbol reversal}

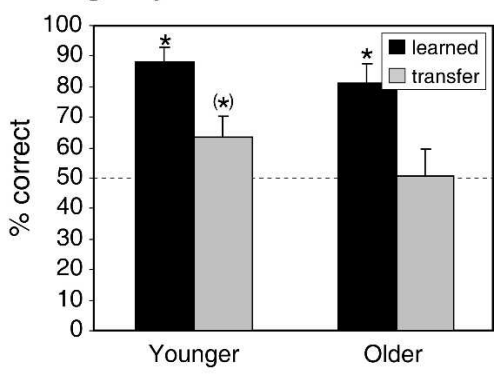

B Acquired equivalence

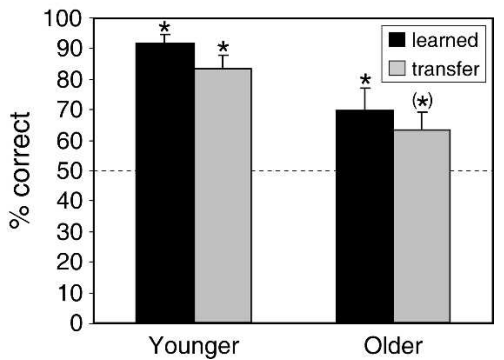

Figure 4. Results of the test stages of the single symbol reversal phase of task $1(A)$ and the acquired equivalence task $(B)$. Dashed lines indicate the chance level; asterisks denote performance significantly above chance. $\left(^{*}\right) 0.05<P<0.10$. Error bars represent standard errors. 
Table 2. Response times

\begin{tabular}{lccccccc}
\hline & \multicolumn{3}{c}{ Acquisition } & & \multicolumn{3}{c}{ Reversal } \\
\cline { 2 - 3 } & Block 1 & Block 2 & Block 3 & & Block 1 & Block 2 & Block 3 \\
\hline Young & $885(216)$ & $804(235)$ & $780(279)$ & & $766(288)$ & $773(267)$ & $758(263)$ \\
Old & $1043(268)$ & $996(271)$ & $1007(313)$ & & $909(242)$ & $887(269)$ & $873(212)$ \\
\hline
\end{tabular}

Mean RTs (SDs in brackets) in milliseconds for acquisition and reversal.

equivalence findings were limited to the subgroup of learners. On a conventional declarative learning task that did not involve rewards (verbal paired associates), overall performance was reduced in older compared to younger subjects, in particular for pairs that were not semantically related, at least during the initial learning stages. Similar to acquisition during reward learning, there was significant learning across blocks in the older group, reaching the level of younger subjects on the third block. This effect may, however, be due to ceiling effects in the younger group.

The older subjects of this study had completed fewer years of education than the younger subjects, which is clearly a cohort effect linked to the limited possibilities for higher education in the generation born in the 1930s and 1940s in Germany. This education difference cannot convincingly explain the observed pattern of results on the learning tasks. Although completing fewer years of education, older participants achieved higher present-state IQ estimates than younger subjects, showing that a lower number of years of education did not lead to lower general intellectual abilities. The difference in IQ (5 points) was too small to have a significant effect on the outcome of the learning tasks. Additionally, if there were an effect of IQ on learning abilities in the current experiment, the higher IQ should be of advantage for the older participants rather than contributing to their reward learning difficulties. Furthermore, exploratory correlational analysis of reward learning measures and IQ did not yield any significant correlations in any of the age groups (all $r<0.3$, $P>0.1$ ). Short-term memory abilities, on the other hand, might affect learning, since memorizing of the Asian symbols in the reward learning tasks requires maintenance, yet no active manipulation of the relevant stimuli, that is, did not recruit working memory abilities. Short-term memory measures did not, however, correlate significantly with the learning measures. In the older group, visuospatial working memory correlated significantly with acquisition, although the correlation was relatively low $(r=0.5)$. Importantly, effects of reward magnitude and reversal learning measures were unrelated to short-term and working memory. Along similar lines, Mell et al. (2005) reported that age differences in working memory performance could not explain reversal learning impairments of the older subjects. It is also noteworthy that in the present study only visuospatial, but not verbal short-term and working memory, differed significantly between the groups, and post-experimental interviews revealed that the majority of subjects used a verbal strategy to facilitate memory of the Asian symbols. Evaluation of the postexperimental interviews also indicated that older subjects rated their ability to discriminate between the four symbols as poorer than the young subjects. Statistical analysis limited to subjects who gave high ratings of their recognition abilities of the symbols (scores 1 or 2 on a 5 -point scale, 5 being the lowest) replicated the group differences between younger and older groups. Taken together, it is thus unlikely that age differences in general memory abilities can fully explain age effects in reward-based learning.

Aging is accompanied by a general loss of dopamine receptors and transporters in striatal and extrastriatal regions (Bäck- man et al. 2006), which is likely to adversely affect reward-based learning abilities. In addition, striatal nuclei (caudate nucleus and putamen), which play a key role in reward-based associative learning, undergo pronounced volume shrinkage over the life span (Murphy 1985; Haug and Eggers 1991; GunningDixon et al. 1998; Raz et al. 2003). Although no direct conclusions about neural age-related changes can be drawn from our purely behavioral results concerning reward-based learning, the observed impairments are in line with changes reported in the literature. The older subjects of the present study were impaired in reward-based associative learning, exhibiting a smaller number of correct responses and needing more trials to reach the learning criterion. Remarkably, this deficit pattern was observed in almost all parts of the learning tasks, even during acquisition of the initial stimulus contingencies, that is, before the more demanding reversal learning phase. This appears to be inconsistent with findings reported by Fera et al. (2005), who found equivalent performance of younger and older subjects on the weather prediction task. However, there is an important difference between the tasks: Fera et al. (2005) addressed performance feedback-based associative learning, whereas we used a reward-based learning procedure. More precisely, in contrast to other reward learning studies (e.g., Mell et al. 2005), subjects in the present study were not paid a fixed amount of money but earned their cumulative gains based on their own performance; that is, each reinforced trial contained a "real" reward. However, a recent category classification learning study also reported age-related impairments in a performance feedback-based but not in an observational version of their task, which is in agreement with our findings, although there are considerable differences with respect to stimulus material, task design, and type of feedback (Schmitt-Eliassen et al. 2007).

The results of the present study are in good agreement with the reversal learning deficits observed in animal aging studies (Lai et al. 1995; Herndon et al. 1997; Voytko 1999; Schoenbaum et al. 2002; Tapp et al. 2003) and with previous findings of stimulus-reward association learning in human subjects (Mell et al. 2005). While Mell et al. (2005) focused on reversal of contingencies using a stimulus-reward learning task, the present study extends aging effects on reversal learning to a more complex type of reward-based stimulus-stimulus association learning and additionally to the slow initial acquisition phase. Although we cannot clearly distinguish between different types of errors underlying the nature of the older adults' impairments, our data do not favor perseveration as their underlying cause, since performance started on a relatively high level during reversal and clearly not below $50 \%$, as would be expected from the consistent application of the previously correct strategy. Reversal learning is commonly linked to the OFC in animals (e.g., Chudasama and Robbins 2003; Izquierdo et al. 2004) and humans (Rolls et al. 1994; Fellows and Farah 2003; Hornak et al. 2004). The degree of agerelated degenerative changes in the human OFC is still a matter of debate: While Raz et al. (1997) found age-related volume loss in OFC, others reported preservation of or even an increase in OFC volume with age (Salat et al. 2001, 2002), and the exact findings may depend on the methods used to assess OFC volume (Tisserand et al. 2002). Also the dorsolateral PFC and the amygdala have been implicated in the mediation of the complex processes underlying reversal learning (Hornak et al. 2004; Stalnaker et al. 2007). There are consistent reports of declining lateral PFC volume with age (Tisserand et al. 2002; Hedden and Gabrieli 2004; Raz et al. 2005), which is in accordance with behavioral 
results of MacPherson et al. (2002), who found age-related impairments in tasks tapping dorsolateral PFC function but did not observe age effects in tests of ventromedial PFC functions, although some of the latter results (Iowa Gambling Task) were challenged by Denburg et al. (2005). The impairments of the older adults in the present study may be at least partly related to PFC dysfunction. Evidence for reduced frontal function of our older participants stems from the observed working memory impairments on the block span task and the large difference in paired associate learning for easy and hard items (see Fig. 2), which resembles the pattern observed in frontal lesion patients (Daum et al. 1995).

Unlike reversal learning, the processing of different reward magnitudes seemed to be preserved with age since both groups showed better overall acquisition for high compared to low reward magnitudes. However, when only those subjects who showed evidence of significant learning were considered, learning was similarly fast with 5 and 20 cent rewards in young subjects, whereas older subjects appeared to learn slower with low compared to high reward magnitudes. The reward learning theory assumes that learning only occurs if a prediction error is present, that is, if there is an incongruity between actual and expected rewards (e.g., Schultz 2002). In trials rewarded with 20 cents, in monetary terms the prediction error was larger compared to trials rewarded with 5 cents. It could be speculated that older subjects might require a larger prediction error for fast learning, which would explain the differential results for the two reward magnitudes.

In addition to the acquisition and reversal learning problems, older subjects had mild difficulties with transfer learning and generalization: Successful transfer was not observed during single symbol reversal, and there was only a marginal effect in the acquired equivalence test. However, it should be pointed out that transfer performance in the younger subjects also did not significantly exceed the chance level, suggesting a very high difficulty level of the task. To successfully achieve single symbol reversal, participants had to learn the reversal of contingencies and transfer their knowledge to previously unlearned stimuli, presumably requiring activation of MTL and PFC regions (Rolls 2000; Myers et al. 2003; Keri et al. 2005; Kringelbach 2005). For generalization and transfer in the acquired equivalence task, however, reversal (and presumed PFC involvement) was not required. This difference between requirements might explain the observed performance differences between the tasks. The transfer impairments of the older subjects in the acquired equivalence test resemble their difficulties during learning of hard items in the verbal paired associates task. Both tasks presumably rely to some degree on the functional integrity of the MTL, which is generally reduced after the age of 60 (Hedden and Gabrieli 2004).

Because of the probabilistic nature of our tasks, nondeclarative learning of the stimulus contingencies was assumed to play a major role in the initial acquisition and reversal phases, which is also supported by the linear trend shape of the learning curves, at least in the younger subjects. Nevertheless, since only a small set of stimuli (i.e., four Asian symbols and two colors) was used for associative learning, the recruitment of declarative learning mechanisms cannot be completely ruled out. Inspection of post-experimental questionnaire data indicated that at least some subjects had become aware of the contingencies and could verbalize a successful strategy, thereby making use of declarative learning strategies. This was, however, the case for only five young subjects in the initial reward learning task, but for 15 younger subjects and one older subject in the acquired equivalence paradigm. These findings suggest that younger subjects tended to solve the acquired equivalence task by relying more on declarative strategies and hence presumably on MTL functions than older adults. Consistent with this hypothesis, learners performed better than non-learners on the MTL-dependent verbal paired associates task. Interestingly, there were few significant intercorrelations between the different reward-based learning measures, which were limited to measures derived from the same task. Thus, correlational analysis does not provide convincing evidence for the idea that the different tasks rely on the same underlying process.

The discussion of the relationship between reward-based learning deficits and the reduced volume of specific brain regions in older subjects must remain speculative at this stage since we did not include direct measures of brain volume. As Raz and Rodrigue (2006) have pointed out, correlations between volume reduction and functional impairments are generally modest. In addition, compensatory mechanisms, as, for example, the recruitment of additional brain areas, might allow older adults to learn as well as younger people (Cabeza et al. 2002; Lamar et al. 2004; Fera et al. 2005). Nevertheless, the general pattern of findings of age-associated changes in the acquisition of reward-based stimulus-stimulus associations, in the reversal of contingencies, and in cognitive transfer is clearly consistent with current assumptions about the pattern of age-related changes in the human brain.

\section{Materials and Methods}

\section{Participants}

Thirty healthy young (15 female) and 30 healthy older (16 female) human subjects participated in the experiments. The age range of the younger group was 19-33 yr, with a mean age of 24.2 $\mathrm{yr}(\mathrm{SD}=2.8)$; the age range in the older group was $50-71 \mathrm{yr}$, with a mean age of $64.1 \mathrm{yr}(\mathrm{SD}=6.2)$. The two groups differed significantly on years of education and IQ estimates based on the subtests "Picture completion" and "Similarities" of a German version of the Wechsler Adult Intelligence Scale (Dahl 1972). Years of education were lower in the older participants because of cohort effects, but they scored higher on the present-state IQ estimate (see Table 1). Short-term and working memory were assessed by the digit (verbal) and block span (visuospatial) subtests of the Wechsler memory scale (Wechsler 1987).

All participants had normal or corrected-to-normal vision, and they were screened for general health status. Exclusion criteria were history of psychiatric or neurological disease and regular use of any medication affecting the central nervous system. All but one of the young subjects were right-handed. The study was approved by the Ethics Committee of the Medical Faculty of the Ruhr-University of Bochum, and each subject signed an informed consent form. The subjects were paid a minimum of 20 euro to reimburse travel and other expenses related to participation. Depending on their performance in the learning tasks, they could increase this sum (see below).

\section{Procedure}

After completion of the IQ and working memory tasks, three tests of associative learning were administered. The verbal paired associates subtest of the Wechsler Memory Scale (Wechsler 1987) was conducted to assess declarative learning associated with MTL function. Eight word pairs are read to the subject. The first word of each pair is then read, and the subject has to reproduce the corresponding second word from memory. There are four easy pairs (e.g., metal/iron) and four hard pairs (e.g., salad/pencil), and learning is repeated twice. Following paired associates learning, the reward-based learning tasks were administered. The first task was preceded by a short practice session, in which subjects could familiarize themselves with the procedure. Stimuli were presented on an Eizo FlexScan F56 color monitor using Presentation 9.90. The testing session lasted for $\sim 2 \mathrm{~h}$. 


\section{Stimulus-reward learning: Acquisition, reversal, and single symbol reversal}

Details of the task are depicted in Figure 1. Subjects had to learn the association between four Asian symbols (Fig. 1B) and two colors (red and green) on the basis of feedback provided after each trial. Before the start of the experiment, it was made sure that participants could easily distinguish between the colors. Each of the symbols was associated with a particular color, that is, the choice of one color (e.g., red) led to reward, whereas the choice of the other color (e.g., green) led to no reward. Feedback was given in form of an icon of monetary reward, and subjects were informed before the start of the experiment that the sum of their gains would be paid out to them after completion of the tasks.

The learning task consisted of three phases (see Fig. 1B). In the first phase (acquisition), two of the Asian symbols were associated with "red," the remaining two symbols with "green." These symbol-color associations were inverted in the second phase (reversal) so that the formerly "red"-associated symbols now predicted that "green" was the response leading to reward, and vice versa. Acquisition and reversal consisted of 120 trials each (three blocks of 40 trials each).

At the beginning of the third phase (single symbol reversal), the symbol-color associations were changed back to the initial contingencies of the acquisition phase. However, during learning, only two of the symbols were used (one associated with "red," one with "green;" learned symbols). In the subsequent trials (test stage), all four initial symbols were used again, that is, subjects needed to transfer the reversal of contingencies to the symbols not used for new learning (transfer symbols). There was no trial-by-trial feedback in the test stage. Correct responding to all four symbols was thus achieved, if the subject had learned that (1) two symbols had consistently been paired with one color or (2) that the symbol-color associations had been switched back to the contingencies of the acquisition phase. Single symbol acquisition ended after correct responses on five successive trials (minimum of 15 trials) or if this criterion was not reached after 50 trials. The subsequent test stage involved 40 trials. For motivational purposes, subjects were informed about their cumulative gains every five trials.

As illustrated in Figure 1A, each trial started with the presentation of a fixation cross in the center of the screen for $1 \mathrm{sec}$, followed by the display of one of the Asian symbols for $2.5 \mathrm{sec}$. The screen then went black for $1 \mathrm{sec}$, followed by the presentation of a white, a red, and a green circle horizontally aligned. While the positions of the red and green circles were randomized over the trials, the white circle always appeared in the center. The subject was instructed to select either the red or the green circle by pressing one of two keys within a time window of $3 \mathrm{sec}$. Following the button press, the color of the chosen circle changed to white to confirm the subject's selection $(0.5 \mathrm{sec})$. After presentation of a black screen for $1 \mathrm{sec}$, feedback about the choice was provided. Correct choices were followed by the presentation of an icon of either a 5 or a 20 cent coin on one of three white circles; incorrect choices led to the display of three empty white circles.

Throughout the experiment, the same two symbols were associated with 5 cents, the remaining two symbols with 20 cents. However, because of the probabilistic nature of the task, the subjects received rewards in only $80 \%$ of the trials, that is, correct choices were not always reinforced by a reward. The reward/non-reward sequence of trials was determined randomly, as was the sequence of the presentation of the four symbols with each symbol being used at equal frequency. The symbol-color pairings and the pairings of symbols and reward magnitudes (5 or 20 cents) were counterbalanced across subjects.

\section{Acquired equivalence}

The probabilistic acquired equivalence task involved associations between four Asian symbols and two colors as in the task described above. The structure of each trial was also identical to the first experiment (see Fig. 1A); the reward magnitude was, how- ever, always 5 cents, and new Asian symbols (Fig. 1C) and colors were used. Trials with different symbols were randomized, and the symbol-color association patterns were counterbalanced across subjects.

The acquired equivalence task consisted of three phases (see Fig. 1C). During the initial phase (acquisition phase 1), participants had to learn based on monetary feedback that two of the Asian symbols (symbols 1 and 2) were associated with the color pink and the other two (symbols 3 and 4) were associated with the color brown. Thus, symbols 1 and 2 were equivalent with respect to the associated color, as were symbols 3 and 4 . The reward probability was $80 \%$. Acquisition was completed when the subjects either reached a criterion of eight successive correct responses (minimum of 38 trials) or after 80 trials. In the subsequent acquisition phase 2 , the colors changed (blue and yellow), and only two of the four symbols (symbols 1 and 3) were used. Symbol 1, which had previously been associated with the color pink, now predicted that "blue" was the correct response; symbol 3 , which had previously been associated with the color brown, now indicated that "yellow" was the correct response. In the third phase (test phase), the newly learned associations between the new colors and symbols 1 and 3 had to be transferred to the other two symbols. The test phase started after five correct responses in a row (minimum of 15 trials) or after 80 trials, if this criterion was not reached. The 40 trials of the test phase involved all four symbols, and there was no trial-by-trial feedback. Subjects were, however, informed about their cumulative gains every five trials. Hence, correct responses to all symbols could be achieved if the subjects had learned the equivalence of symbols 1 and 2 and symbols 3 and 4 , respectively, during acquisition.

\section{Statistical analysis}

Statistical analyses were performed using SPSS 15.0. Repeated measures data sets were analyzed by means of analysis of variance or $t$-tests, where appropriate. The significance level was set at 0.05 (two-tailed). All trials, in which the correct color was chosen, whether they were rewarded or not owing to the probabilistic setup, were considered as correct trials and included in the analyses. Trials following rewarded correct trials were treated the same way as trials following non-rewarded correct trials.

\section{Acknowledgments}

This research was supported by the German Ministry of Education and Research (BMBF, grant no. 01 GW0541) and by the RuhrUniversity Research School funded by the German Research Society (DFG) in the framework of the Excellence Initiative.

\section{References}

Aron, A.R., Shohamy, D., Clark, J., Myers, C., Gluck, M.A., and Poldrack, R.A. 2004. Human midbrain sensitivity to cognitive feedback and uncertainty during classification learning. J. Neurophysiol. 92: 1144-1152.

Bäckman, L., Nyberg, L., Lindenberger, U., Li, S.C., and Farde, L. 2006. The correlative triad among aging, dopamine, and cognition: Current status and future prospects. Neurosci. Biobehav. Rev. 30: 791-807.

Beninger, R.J. and Gerdjikov, T. 2004. The role of signaling molecules in reward-related incentive learning. Neurotox. Res. 6: 91-104.

Cabeza, R., Anderson, N.D., Locantore, J.K., and McIntosh, A.R. 2002. Aging gracefully: Compensatory brain activity in high-performing older adults. Neuroimage 17: 1394-1402.

Chudasama, Y. and Robbins, T.W. 2003. Dissociable contributions of the orbitofrontal and infralimbic cortex to Pavlovian autoshaping and discrimination reversal learning: Further evidence for the functional heterogeneity of the rodent frontal cortex. J. Neurosci. 23: $8771-8780$

Dahl, G. 1972. WIP-Reduzierter Wechsler Intelligenztest: Anwendung, Auswertung Statistische Analysen, Normwerte. Hain, Meisenheim, Germany.

Daum, I., Schugens, M.M., Spieker, S., Poser, U., Schönle, P.W., and Birbaumer, N. 1995. Memory and skill acquisition in Parkinson's disease and frontal lobe dysfunction. Cortex 31: 413-432.

Delgado, M.R. 2007. Reward-related responses in the human striatum. Ann. N. Y. Acad. Sci. 1104: 70-88. 
Delgado, M.R., Nystrom, L.E., Fissell, C., Noll, D.C., and Fiez, J.A. 2000 Tracking the hemodynamic responses to reward and punishment in the striatum. J. Neurophysiol. 84: 3072-3077.

Delgado, M.R., Locke, H.M., Stenger, V.A., and Fiez, J.A. 2003. Dorsal striatum responses to reward and punishment: Effects of valence and magnitude manipulations. Cogn. Affect. Behav. Neurosci. 3: 27-38.

Denburg, N.L., Tranel, D., and Bechara, A. 2005. The ability to decide advantageously declines prematurely in some normal older persons. Neuropsychologia 43: 1099-1106.

Elliott, R., Friston, K.J., and Dolan, R.J. 2000. Dissociable neural responses in human reward systems. J. Neurosci. 20: 6159-6165.

Elliott, R., Newman, J.L., Longe, O.A., and Deakin, J.F. 2003. Differential response patterns in the striatum and orbitofrontal cortex to financial reward in humans: A parametric functional magnetic resonance imaging study. J. Neurosci. 23: 303-307.

Fellows, L.K. and Farah, M.J. 2003. Ventromedial frontal cortex mediates affective shifting in humans: Evidence from a reversal learning paradigm. Brain 126: 1830-1837.

Fera, F., Weickert, T.W., Goldberg, T.E., Tessitore, A., Hariri, A., Das, S., Lee, S., Zoltick, B., Meeter, M., Myers, C.E., et al. 2005. Neural mechanisms underlying probabilistic category learning in normal aging. J. Neurosci. 25: 11340-11348.

Gunning-Dixon, F.M., Head, D., McQuain, J., Acker, J.D., and Raz, N. 1998. Differential aging of the human striatum: A prospective MR imaging study. AJNR Am. J. Neuroradiol. 19: 1501-1507.

Haug, H. and Eggers, R. 1991. Morphometry of the human cortex cerebri and corpus striatum during aging. Neurobiol. Aging 12: $336-338$.

Hedden, T. and Gabrieli, J.D. 2004. Insights into the ageing mind: A view from cognitive neuroscience. Nat. Rev. Neurosci. 5: 87-96.

Herndon, J.G., Moss, M.B., Rosene, D.L., and Killiany, R.J. 1997. Patterns of cognitive decline in aged rhesus monkeys. Behav. Brain Res. 87: $25-34$

Hornak, J., O'Doherty, J., Bramham, J., Rolls, E.T., Morris, R.G., Bullock, P.R., and Polkey, C.E. 2004. Reward-related reversal learning after surgical excisions in orbito-frontal or dorsolateral prefrontal cortex in humans. J. Cogn. Neurosci. 16: 463-478.

Izquierdo, A., Suda, R.K., and Murray, E.A. 2004. Bilateral orbital prefrontal cortex lesions in rhesus monkeys disrupt choices guided by both reward value and reward contingency. J. Neurosci. 24: $7540-7548$.

Keri, S., Nagy, O., Kelemen, O., Myers, C.E., and Gluck, M.A. 2005 Dissociation between medial temporal lobe and basal ganglia memory systems in schizophrenia. Schizophr. Res. 77: 321-328.

Knowlton, B.J., Mangels, J.A., and Squire, L.R. 1996. A neostriatal habit learning system in humans. Science 273: 1399-1402.

Knutson, B., Taylor, J., Kaufman, M., Peterson, R., and Glover, G. 2005. Distributed neural representation of expected value. J. Neurosci. 25: $4806-4812$.

Kringelbach, M.L. 2005. The human orbitofrontal cortex: Linking reward to hedonic experience. Nat. Rev. Neurosci. 6: 691-702.

Lai, Z.C., Moss, M.B., Killiany, R.J., Rosene, D.L., and Herndon, J.G. 1995. Executive system dysfunction in the aged monkey: Spatial and object reversal learning. Neurobiol. Aging 16: 947-954.

Lamar, M., Yousem, D.M., and Resnick, S.M. 2004. Age differences in orbitofrontal activation: An fMRI investigation of delayed match and nonmatch to sample. Neuroimage 21: 1368-1376.

Larkin, G.R., Gibbs, S.E., Khanna, K., Nielsen, L., Carstensen, L.L., and Knutson, B. 2007. Anticipation of monetary gain but not loss in healthy older adults. Nat. Neurosci. 10: 787-791.

MacPherson, S.E., Phillips, L.H., and Della Sala, S. 2002. Age, executive function, and social decision making: A dorsolateral prefrontal theory of cognitive aging. Psychol. Aging 17: 598-609.

Marschner, A., Mell, T., Wartenburger, I., Villringer, A., Reischies, F.M., and Heekeren, H.R. 2005. Reward-based decision-making and aging Brain Res. Bull. 67: 382-390.

Mell, T., Heekeren, H.R., Marschner, A., Wartenburger, I., Villringer, A., and Reischies, F.M. 2005. Effect of aging on stimulus-reward association learning. Neuropsychologia 43: 554-563.

Murphy Jr., G.M. 1985. The human corpus striatum and dentate nucleus: Volumetric analysis for hemispheric asymmetries, sex differences, and aging changes. Exp. Neurol. 89: 134-145.

Myers, C.E., Shohamy, D., Gluck, M.A., Grossman, S., Kluger, A., Ferris, S., Golomb, J., Schnirman, G., and Schwartz, R. 2003. Dissociating hippocampal versus basal ganglia contributions to learning and transfer. J. Cogn. Neurosci. 15: 185-193.

O'Doherty, J.P. 2004. Reward representations and reward-related learning in the human brain: Insights from neuroimaging. Curr Opin. Neurobiol. 14: 769-776.

O’Doherty, J.P., Kringelbach, M.L., Rolls, E.T., Hornak, J., and Andrews, C. 2001. Abstract reward and punishment representations in the human orbitofrontal cortex. Nat. Neurosci. 4: 95-102.
Poldrack, R.A., Clark, J., Paré-Blagoev, E.J., Shohamy, D., Creso Moyano, J., Myers, C., and Gluck, M.A. 2001. Interactive memory systems in the human brain. Nature 414: 546-550.

Rapp, P.R. 1990. Visual discrimination and reversal learning in the aged monkey (Macaca mulatta). Behav. Neurosci. 104: 876-884.

Raz, N. and Rodrigue, K.M. 2006. Differential aging of the brain: Patterns, cognitive correlates and modifiers. Neurosci. Biobehav. Rev. 30: $730-748$.

Raz, N., Gunning, F.M., Head, D., Dupuis, J.H., McQuain, J., Briggs, S.D. Loken, W.J., Thornton, A.E., and Acker, J.D. 1997. Selective aging of the human cerebral cortex observed in vivo: Differential vulnerability of the prefrontal gray matter. Cereb. Cortex 7: 268-282.

Raz, N., Rodrigue, K.M., Kennedy, K.M., Head, D., Gunning-Dixon, F., and Acker, J.D. 2003. Differential aging of the human striatum: Longitudinal evidence. AJNR Am. J. Neuroradiol. 24: 1849-1856.

Raz, N., Lindenberger, U., Rodrigue, K.M., Kennedy, K.M., Head, D., Williamson, A., Dahle, C., Gerstorf, D., and Acker, J.D. 2005. Regional brain changes in aging healthy adults: general trends, individual differences and modifiers. Cereb. Cortex 15: 1676-1689.

Roesch, M.R. and Olson, C.R. 2004. Neuronal activity related to reward value and motivation in primate frontal cortex. Science 304: $307-310$.

Rolls, E.T. 2000. The orbitofrontal cortex and reward. Cereb. Cortex 10: $284-294$.

Rolls, E.T., Hornak, J., Wade, D., and McGrath, J. 1994. Emotion-related learning in patients with social and emotional changes associated with frontal lobe damage. J. Neurol. Neurosurg. Psychiatr. 57: 1518-1524.

Salat, D.H., Kaye, J.A., and Janowsky, J.S. 2001. Selective preservation and degeneration within the prefrontal cortex in aging and Alzheimer disease. Arch. Neurol. 58: 1403-1408.

Salat, D.H., Kaye, J.A., and Janowsky, J.S. 2002. Greater orbital prefrontal volume selectively predicts worse working memory performance in older adults. Cereb. Cortex 12: 494-505.

Schmitt-Eliassen, J., Ferstl, R., Wiesner, C., Deuschl, G., and Witt, K. 2007. Feedback-based versus observational classification learning in healthy aging and Parkinson's disease. Brain Res. 1142: 178-188.

Schoenbaum, G., Nugent, S., Saddoris, M.P., and Gallagher, M. 2002. Teaching old rats new tricks: Age-related impairments in olfactory reversal learning. Neurobiol. Aging 23: 555-564.

Schott, B.H., Niehaus, L., Wittmann, B.C., Schutze, H., Seidenbecher, C.I., Heinze, H.J., and Duzel, E. 2007. Ageing and early-stage Parkinson's disease affect separable neural mechanisms of mesolimbic reward processing. Brain 130: $2412-2424$.

Schultz, W. 2000. Multiple reward signals in the brain. Nat. Rev. Neurosci. 1: 199-207.

Schultz, W. 2002. Getting formal with dopamine and reward. Neuron 36: $241-263$.

Shohamy, D., Myers, C.E., Grossman, S., Sage, J., Gluck, M.A., and Poldrack, R.A. 2004. Cortico-striatal contributions to feedback-based learning: Converging data from neuroimaging and neuropsychology. Brain 127: 851-859.

Stalnaker, T.A., Franz, T.M., Singh, T., and Schoenbaum, G. 2007. Basolateral amygdala lesions abolish orbitofrontal-dependent reversal impairments. Neuron 54: 51-58.

Tapp, P.D., Siwak, C.T., Estrada, J., Head, E., Muggenburg, B.A., Cotman, C.W., and Milgram, N.W. 2003. Size and reversal learning in the beagle dog as a measure of executive function and inhibitory control in aging. Learn. Mem. 10: 64-73.

Tisserand, D.J. and Jolles, J. 2003. On the involvement of prefrontal networks in cognitive ageing. Cortex 39: 1107-1128.

Tisserand, D.J., Pruessner, J.C., Sanz Arigita, E.J., van Boxtel, M.P., Evans, A.C., Jolles, J., and Uylings, H.B. 2002. Regional frontal cortical volumes decrease differentially in aging: An MRI study to compare volumetric approaches and voxel-based morphometry. Neuroimage 17: 657-669.

Voytko, M.L. 1999. Impairments in acquisition and reversals of two-choice discriminations by aged rhesus monkeys. Neurobiol. Aging 20: $617-627$.

Wallis, J.D. 2007. Orbitofrontal cortex and its contribution to decision-making. Annu. Rev. Neurosci. 30: 31-56.

Wallis, J.D. and Miller, E.K. 2003. Neuronal activity in primate dorsolateral and orbital prefrontal cortex during performance of a reward preference task. Eur. J. Neurosci. 18: 2069-2081.

Wechsler, D. 1987. Wechsler memory scale-Revised. Psychological Corporation, London.

Zink, C.F., Pagnoni, G., Martin-Skurski, M.E., Chappelow, J.C., and Berns, G.S. 2004. Human striatal responses to monetary reward depend on saliency. Neuron 42: 509-517.

Received December 20, 2007; accepted in revised form January 30, 2008. 


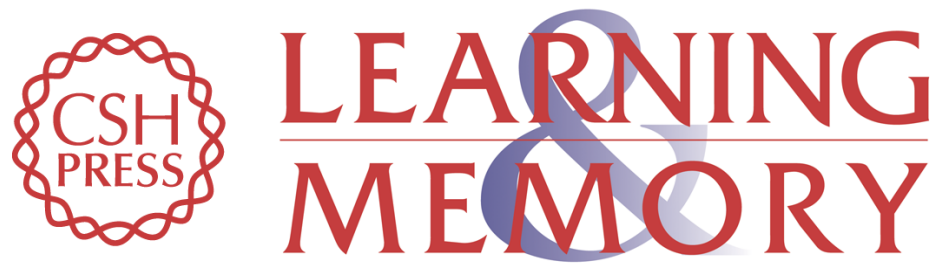

\section{Aging affects acquisition and reversal of reward-based associative learning}

Julia A. Weiler, Christian Bellebaum and Irene Daum

Learn. Mem. 2008, 15:

Access the most recent version at doi:10.1101//m.890408

References This article cites 59 articles, 10 of which can be accessed free at:

http://learnmem.cshlp.org/content/15/4/190.full.html\#ref-list-1

License

Email Alerting Receive free email alerts when new articles cite this article - sign up in the box at the Service top right corner of the article or click here. 